50

\title{
DUALQUEST: An Implementation of the Real-time Bifocal Visualization for Network Management
}

\author{
Shoichiro Nakai, Hiroko Fuji, and Hiroshi Matoba \\ C\&C Research Laboratories, NEC Corporation \\ 1-1, Miyazaki 4, Miyamaeku, Kawasaki 216 JAPAN \\ Tel:+81-44-856-2314, Fax:+81-44-856-2229 \\ E-Mail:nakai@nwk.cl.nec.co.jp,fuji@nwk.cl.nec.co.jp, \\ matoba@mmp.cl.nec.co.jp
}

\begin{abstract}
Most of the current network management systems employ graphic-user-interfaces for the network visualization purposes. These are well suited for both small- and medium-size networks. For a large-size network, hierarchical multi-window-based network visualizations are usually used; however, tracing a long path (i.e., composed of a huge number of nodes) may meet some difficulties because it must be at first divided into several segments displayed segment-by-segment in several windows. In addition, window manipulations, such as opening and closing operations, are quite complex. To overcome the disadvantages of the multi-window network visualization, we proposed a real-time bifocal network visualization that is capable of displaying both the context and all details of a network within a single window (Fuji, 1994). This paper enhances that approach and describes an implementation, called DUALQUEST, that was installed in a workstation equipped with a frame buffer memory proposed in (Matoba, 1990) for real-time bifocal image processing.
\end{abstract}

\section{Keywords}

Graphic-user-interface, Network visualization, Bifocal display, Fish-eye view 


\section{INTRODUCTION}

At present, graphic-user-interfaces are widely used to facilitate realization of network management functions. For example, NMS (Network Management System) reports a change of network status to the operators by alternating visual attributes of the graphic symbols displayed on a monitor screen (Cunningham, 1992). If the size of a managed network increases, more symbols must be displayed on the same screen. With regard to this, the hierarchical multi-window presentation (see Figure 1) was proposed. (Hewlett Packard, 1992) Although it is well suited for tracing paths composed of several nodes, paths comprising a huge number of nodes can only be traced on a segment-by-segment basis. This requires passing through several (separate) windows to trace such a path from its origin node to its destination node. Furthermore, the effect of overlapping windows may cause missing some important information. Thus, operators must perform many complex (opening, moving, and closing) window operations to obtain the desired information.

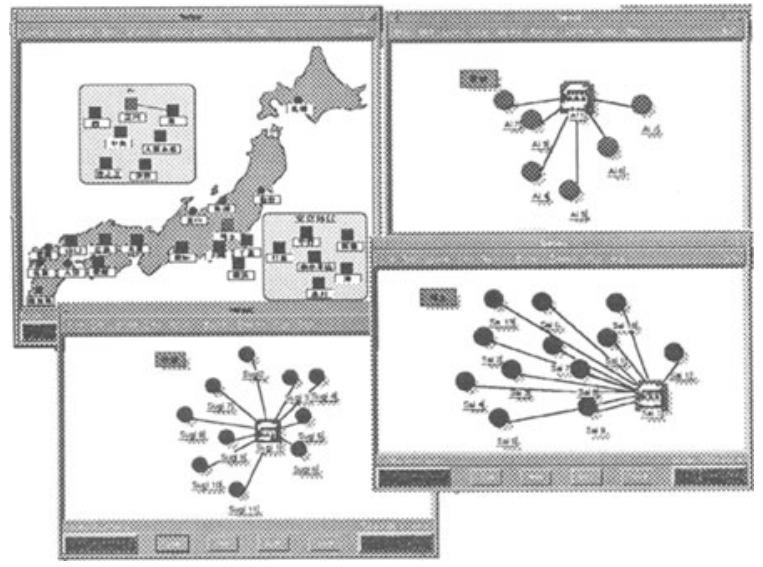

Figure 1 Multi-window graphic-user-interface style.

To overcome those difficulties, we proposed (Fuji, 1994), an approach that uses a bifocal display for providing both the network's context and details within a single window. This paper describes an implementation of it. The implementation, called DUALQUEST, was installed in a workstation equipped with a frame buffer memory for real-time bifocal image generation. For the performance evaluation and comparison purposes, we tested (with the aid of an event simulation program) both DUALQUEST and the hierarchical multi-window presentation in the presence of network alarms caused by, for instance, network element failures. 
The paper is organized as follows. At first, we present the bifocal network visualization and compare it with the hierarchical multi-window visualization (Section 2). Next, DUALQUEST is introduced (Section 3). Then, we describe an experiment that was done to examine performance of those two methods (Section 4). Finally, we discuss some results obtained in the experiment.

\section{BIFOCAL PRESENTATION VS. MULTI-WINDOW PRESENTATION}

Hierarchical multi-window presentations are often used to handle networks which are too large to be meaningfully displayed within a single window. In the approach proposed in (Hewlett Packard, 1992), the complete topology of a managed network is displayed within a single window, while details of the network can be displayed within other windows. This may result in some difficulties for the operator; two of them are now briefly discussed.

Since multiple windows overlap each other, an amount of information can be lost. If a significant information is lost, a network operator must perform complex maneuvers to recover it. Another problem appears when the operator is going to trace a network path that comprises a large number of nodes because a single window displays only one segment of the path. Thus, the operator must monitor several windows to recognize such a path.

To display a large amount of data within a limited area, the bifocal display approach was proposed and analyzed in (Leung, 1989., Sarkar, 1992., or Brown, 1993). For instance, according to (Leung, 1989), a single window covers nine distinct regions as was shown in Figure 2; at any time, one of those regions can be enlarged while the others must be compressed to accommodate the enlargement. This is illustrated in Figure 2, the area ' $\mathrm{a}$ ' is enlarged to the area 'A,' while 'b,' 'c' and 'd' are compressed to 'B,' 'C' and 'D,' respectively. As it is shown in Figure 2, a bifocal image can be generated by combining the data obtained from four different types of images (Misue, 1989).

Advantages of the bifocal approach can be summarized as follows.

- Since views are generated through expanding one area and compressing the others, no objects are missed at any time.

- Since all objects are viewed continuously, the whole nine regions can be easily traversed.

These advantages make bifocal display attractive for a network management user interface. Since, at any time, every object is displayed in a single window, the operator can continuously monitor the status of all network elements. In addition, the operator can traverse network connections displayed in several regions. This plays a key role especially for node-to-node connection management. 


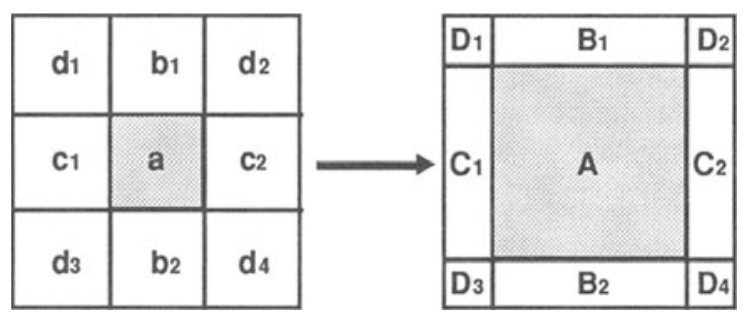

Figure 2 Illustration of the bifocal image generation.

\section{DUALQUEST}

In the bifocal display applications for network management purposes, such as fulfilling the alarm surveillance task, any area should be simply enlarged by clicking the mouse at an appropriate point on the screen. Since real-time responding to network notifications and operator's actions is required, we proposed the real-time bifocal network visualization using a frame buffer memory (Fuji, 1994). Then, the idea has been enhanced and resulted in an implementation, here called DUALQUEST.

\subsection{Rearrangement of network nodes}

Displays of a major city network usually contain many overlapping nodes and links; see, for instance, Figure $3 \mathrm{a}$. To eliminate the overlapping effect and to use a screen more efficiently, a rearrangement of network nodes is required (see Figure $3 \mathrm{~b}$ ).

\subsection{Presentation guideline}

To determine network topology information that should be provided by the bifocal display, a presentation guideline is needed. Generally, two types of network views can be provided by DUALQUEST: the initial view and the enlarged view. To simplify an information display, both node names and link symbols corresponding to the local communication lines are not included to the initial view but they appear within the enlarged view that is, a view generated by the bifocal display using a frame buffer memory. As a result,

- every node name, and 


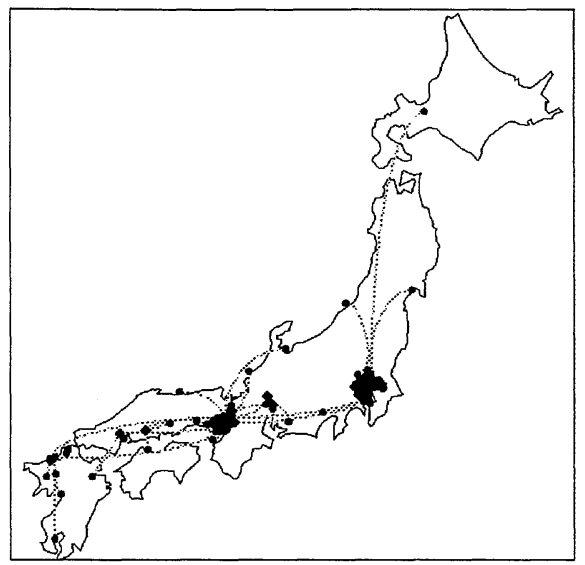

(a) Network before rearrangement

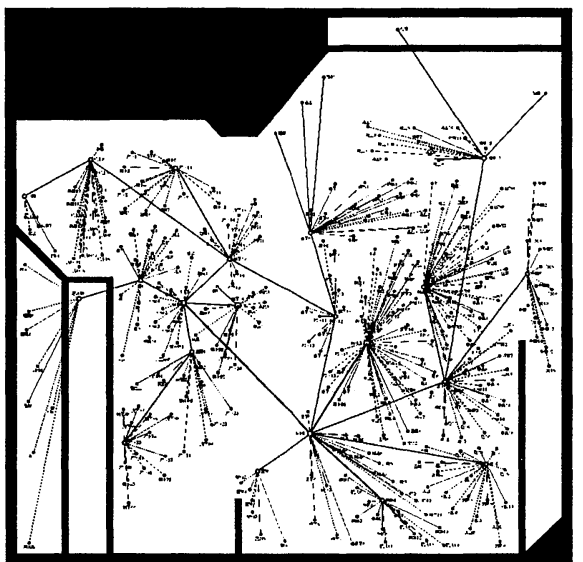

(b) Network after rearrangement

Figure 3 Rearranging network nodes.

- all network connections, including both backbone and local lines, are given in the detailed section of the enlarged view.

Figure 4 shows an example in which the names of nodes and node connections are displayed in detail. In the bifocal network visualization, operators can continuously monitor the status of all network elements in a single window only. In addition, since picture continuity is maintained, network connections are displayed in full detail and the operator can easily trace them. Currently, the full network display is achieved with $900 \times 900$ pixels, while any individual section, displayed with $300 \times 300$ pixels in the initial view, may be enlarged to $600 \times 600$ pixels in the enlarged view.

\subsection{Real-time bifocal image generation}

DUALQUEST is equipped with a frame buffer memory that enables generating bifocal images in real-time. The frame buffer memory is provided with five planes: four image planes, for storing image data, and one plane, for the buffer control (Matoba, 1990). Every pixel-space of the buffer control plane contains address of the image plane whose data should be represented by an appropriate pixel of the bifocal image generated. The bifocal image consists of nine distinct regions; each of them is demarcated in the buffer control plane. Since regions of the same character are characterized by the same magnification (see Figure 2), it is possible to generate a 


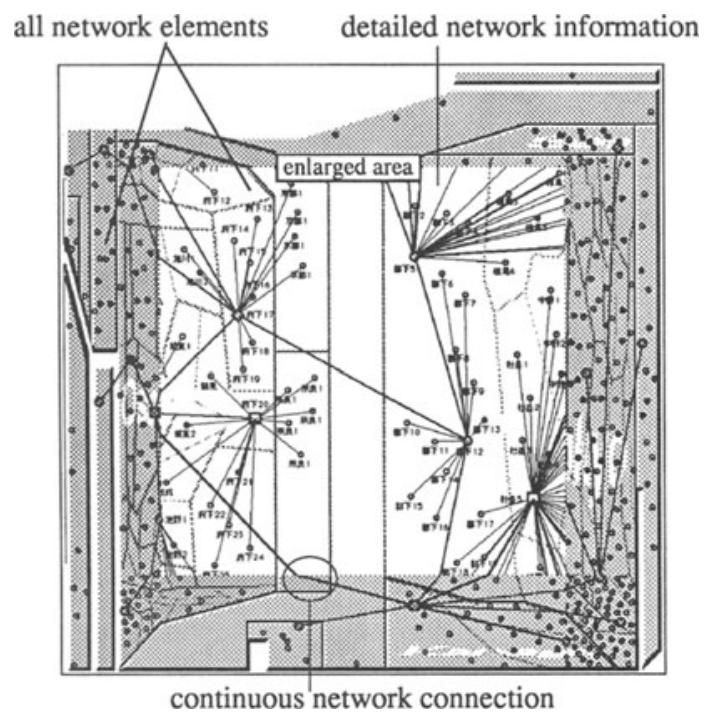

Figure 4 Bifocal network visualization.

bifocal image with only four types of image. Thus, as depicted in Figure 5, a complete bifocal image can be constructed by combining the data of the enlarged image ' $\mathrm{A}$ ' with those of the images 'B,' 'C, 'D,' ..., and 'I' of the three compressed peripheral images. Every pixel-space of the buffer control plane is given by the address of an appropriate image plane. According to the previously described presentation guideline, the enlarged image includes complete information of a network topology, while the compressed peripheral images exclude node names and local lines.

Because all the above operations for bifocal image generation are done in hardware, they can be accomplished instantly at each mouse click. As compared to a software operation, here no computation time is required. Due to this, users can easily traverse network topology as well as they can continuously trace paths of any length. In addition, a larger number of events can be processed within the same period of time since the saved computational time can be spent for fulfilling another task(s).

The current version of DUALQUEST supports fulfilling the alarm surveillance task in a similar way as that described in (Cunningham, 1992); the steps are as follows.

- If an alarm occurs, some symbols corresponding to nodes or links start blinking.

- By making click on the point of interest, the surrounding area appears within the enlarged view.

- The operator can observe the status of all events in a detailed area and follow any 
change, such as back-up, or recovery, of it. This is simply indicated by changing symbol's color.

In fulfilling the alarm surveillance task, the operator can perform such operations as back and forth changes between both the initial and the enlarged views or a change in location of the detailed section. Since all areas remain still visible on the original display, the operator is able to detect other alarms that occur in the compressed network areas and then observe them in more detail. This is illustrated in Figure 6, where at first the surrounding area ' $\mathrm{A}$ ' is enlarged (see Figure 6a), and then the surrounding area ' $B$ ' is enlarged (see Figure $6 \mathrm{~b}$ ). Even then when the detailed section is being focused on the area 'A,' alarms within the area 'B' can be noticed at the time of their occurrence. Thus, all alarms can be seen in those enlarged views; however, some of them may be shrunk in the compressed view.

\section{EXPERIMENT}

To compare the real-time bifocal network presentation (DUALQUEST) with the hierarchical multi-window presentation, we conducted an experiment similar to that proposed in (Mayhe, 1992) for evaluating window style. We selected a sample network, comprising 400 nodes, and an event simulation program that controls the time interval $(5,10$, or 15 seconds) between two consecutive events. Then we invited ten users, including 5 people having no experience with network management systems, to take part in the experiment. Their goal was to fulfill the alarm surveillance tasks by using both the multi-window presentation and DUALQUEST. Operations performed by those users were simultaneously recorded by (i) video cameras, (ii) an eye-markrecorder tracing any movement of the human eye-sight, and (iii) a device sampling mouse operations. All those participants were asked to fill out survey forms twice; before the experiment was started and after it was completed.

\subsection{Multi-window presentation system}

To implement the multi-window presentation system, we used HP OpenView (Hewlett Packard, 1992). In this implementation, we categorized the sample network into 28 groups, where each group aggregated from 10 to 18 nodes. As a result, a two-layer presentation system was built; that is, the network was presented by using 28 group symbols in one window (corresponding to the first layer), while the details of any group were displayed in the second window (corresponding to the second layer). Each of these windows could be opened by clicking the appropriate group symbol on the first layer window. An example window layout in this multiwindow presentation system is illustrated in Figure 1. 
(a) enlarged

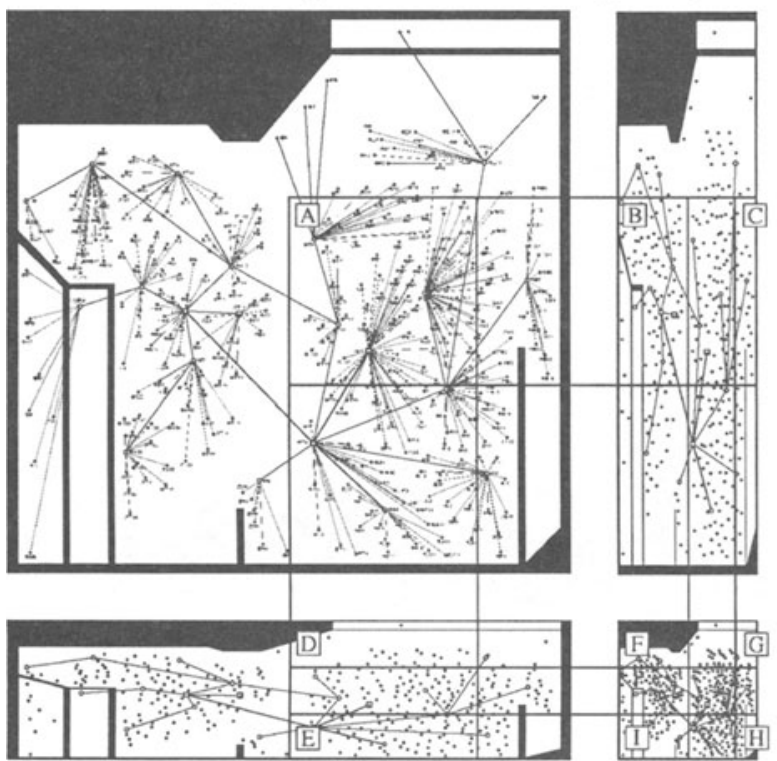

(c) compressed in y (b) compressed in $x$

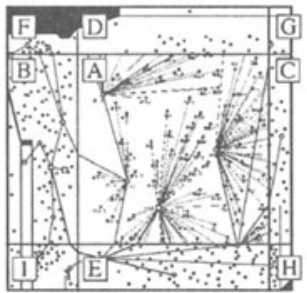

(e) constructed bifocal image

Figure 5 Bifocal image generation using frame buffer memory

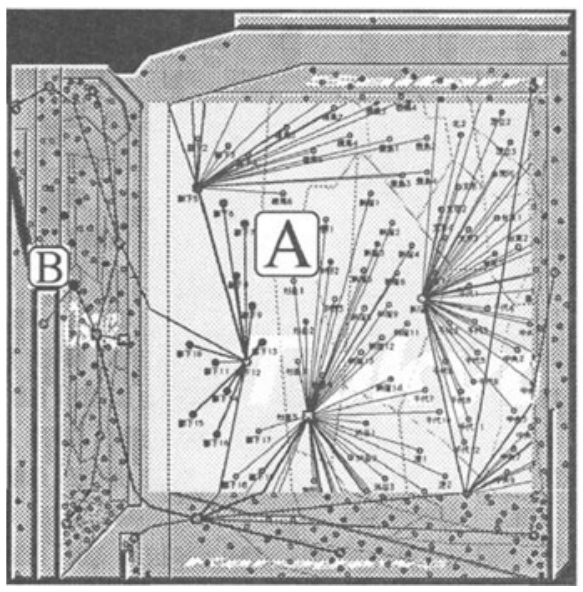

(a) Area 'A' enlarged, area 'B' unenlarged

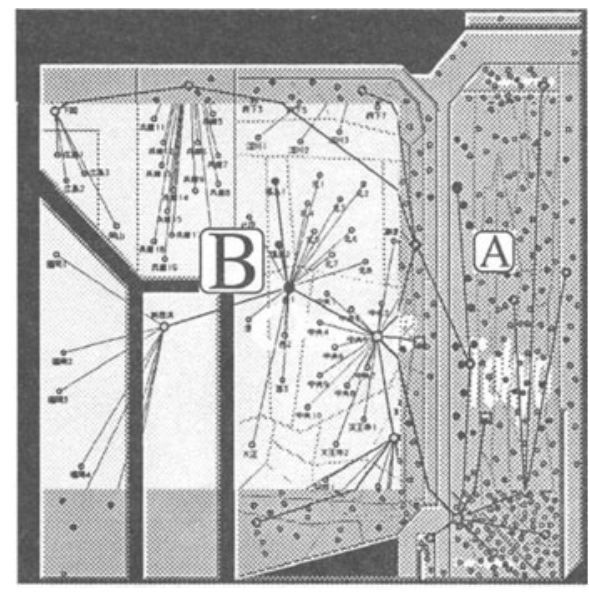

(b) Area 'A' unenlarged, area 'B' enlarged

Figure 6 Screen examples of alarm surveillance. 


\subsection{Results}

As a result of the experiment, DUALQUEST was slightly better than the multi-window presentation system in terms of the time necessary to detect an alarm and the number of alarms not detected within the assumed period of time. For instance Table 1 gives results obtained for the 5 second slots. We think the lack of a performance significant difference between the tested systems was mainly caused by using only two layers of the hierarchical multi-window presentation system.

The two other major results from the experiment can be summarized as follows.

- Nine from the ten users of the ten, among them all inexperienced users, reported finding it easier to discover alarms on DUALQUEST because they were able to perform their tasks in a single window only without complex window operations. (However their first impression on DUALQUEST was not a positive one, since they are used to multi-window style GUI.)

- Smaller windows seem to be more suitable than the whole screen for detecting alarms at the first stage of the alarm surveillance.

The former confirms that even an inexperienced user can operate DUALQUEST, the latter suggests us introducing some user opinions in the further work on DUALQUEST.

Table 1 Experimental results.

\begin{tabular}{lll}
\hline Metric & DUALQUEST & Multi-window System \\
\hline Mean time to & 4.56 seconds & $\begin{array}{l}4.81 \text { seconds } \\
\text { identify all failed nodes }\end{array}$ \\
\hline Mean rate of oversight** & $12 \%$ & 2.03 seconds* \\
\hline$*$ standard deviation & $17 \%$ \\
$* *$ ratio of undetected alarms to all displayed alarms &
\end{tabular}

\section{CONCLUSION}

An implementation (DUALQUEST) of the bifocal display concept in network management systems has been presented and discussed. Since the display is generated by a hardware system using a frame buffer memory, DUALQUEST provides the real-time image. The bifocal display allows the operator to follow all status changes by monitoring a single window on the screen. This advantage of DUALQUEST was confirmed by participants of an experiment that was done to compare the new system with the conventional (the hierarchical multi-window presentation) one. 


\section{Acknowledgment}

The authors wish to thank Y. Hara of NEC Corp. for his technical support and discussion, and would like to give our special thanks to M. Yamamoto, S. Hasegawa, and H. Okazaki, all of NEC Corp. for the encouragement.

\section{REFERENCES}

Brown, H.M., Meehan, R.J. and Sarkar, M. (1993) Browsing Graphs using a Fish-eye View. In proceedings of ACM INTERCHI'93.

Cunningham, P.J., Rotella, J.P., Asplund, L.C., Kawano, H., Okazaki, T., and Mase, K. (1994) Screen Symbols for Network Operations and Management. In proceedings of the Third of Network Operation and Management Symposium.

Fuji, H., Nakai, S., Matoba, H., and Takano, T. (1994) Real-time Bifocal Network Visualization. In proceedings of the Forth of Network Operation and Management Symposium.

Hewlett Packard. (1992) HP OpenView Windows User's Guide. Manual Part Number: J213690000.

Leung, K.Y. (1989) Human-computer Interface Techniques for Map Based Diagrams. In proceedings of the Third International Conference on Human-Computer Interaction.

Matoba, H., Hara, Y. and Kasahara, Y. (1990) Regional Information Guidance System based on Hypermedia Concept. SPIE Vol. 1258 Image Communications and Workstations.

Misue, K. and Sugiyama, K. (1989) A method to display the whole and detail in one figure. 5th Symposium on Human Interface.

Sarkar, M. and Brown, H.M. (1992) Graphical Fish-eye Views of Graphs. In proceedings of ACM SIGCHI'92 Conference on Human Factors In Computing Systems.

Mayhe, D.J. (1992) Principles and Guidelines in Software User Interface Design.

Shoichiro Nakai received his B.E. and M.E. degrees from Keio University in 1981 and 1983, respectively. He joined NEC Corporation in 1983, and has been engaged in the research and development of local area networks, distributed systems, and network management systems. He is currently Research Specialist in the C\&C Research Laboratories.

Hiroko FUJI received her B.E. degree in mathematics from Kyusyu University in 1990. She joined NEC Corporation in 1990, and has been engaged in research on network management. She is currently working in the $\mathrm{C} \& \mathrm{C}$ Research Laboratories.

Hiroshi Matoba received his B.E. degree in Mathematical Engineering and Instrumentation Physics from Tokyo University in 1985, respectively. He joined NEC Corporation in 1985 , and has been engaged in research and development of graphic acceralators for workstations. He is currently an assistant manager in the C\&C Research Laboratories. 